\title{
INVESTIGACIONES
}

\section{Positividad y Negatividad emocional: influencia en los procesos de aprendizajes}

\author{
Emotional Positivity and Negativity: its influence on learning processes \\ Positividade e negatividade emocional: influência nos processos de aprendizagem
}

\author{
Patricio Pacheco Hernández.
}

Universidad Tecnológica Metropolitana

Av. José Pedro Alessandri 1242, Nuñoa

patricio.pacheco@utem.cl

patricio.pacheco03@inacapmail.cl

\begin{abstract}
RESUMEN
Se presenta un procedimiento de registro de datos basado en el seguimiento en aula de las actividades de alumnos constituidos en equipos, los que se encuentran inmersos en procesos de aprendizajes en actividades experimentales de Física Mecánica. Para el análisis de los datos se emplean diversas herramientas matemáticas que entregan como resultado indicadores numéricos que ligan sus aprendizajes, rendimientos, calidad de nexos relacionales a la transformación de sus emociones. El espectro de variables sometidas a observación y posterior estudio, que es influenciado por la evolución de las emociones de los diferentes equipos de alumnos, también abarca el enfoque tradicional de entrega de información desde el exterior (docente en clase magistral) o desde el interior de cada equipo (habilidades de los alumnos) hasta los materiales didácticos para el aprendizaje que potencian la indagación y la persuasión.
\end{abstract}

Palabras Claves: campos emocionales, dinámica no lineal, conectividad, dinámicas de aprendizaje, aprendizajes significativos.

ABSTRACT
This article presents a data collection procedure based on tracking classroom activities where students, formed into teams, are immersed in learning processes of experimental activities in Mechanical Physics. Various mathematical tools are used for the analysis of the data, which deliver results in numerical indicators linking their learning, performance, quality of relational nexus to the transformation of their emotions. The range of variables under observation and further study, influenced by the evolution of the emotions of the different teams of students, covers from the traditional approach of information delivery from the outside (teaching in lecture) or from the inside of each team (abilities of pupils) to instructional materials that enhance learning inquiry and persuasion.

Key words: emotional fields, nonlinear dynamics, connectivity, dynamics of learning, meaningful learning.

\section{RESUMO}

Apresenta-se um procedimento de registro de dados baseado no segmento das aulas onde os estudantes reúnemse em equipes e se encontram imersos em processos de aprendizagem em atividades experimentais de Física Mecânica. Para a análise dos dados, empregam-se diversas ferramentas matemáticas que fornecem indicadores numéricos que ligam suas aprendizagens, rendimentos, qualidade de ligações relacionais à transformação de suas emoções. O conjunto de variáveis submetidas à observação e posterior estudo, influenciado pela evolução das emoções das diferentes equipes de estudantes, também abrange o enfoque tradicional de entrega de informações desde o exterior (docente em aula universitária) ou a partir do interior de cada equipe (habilidades dos alunos) até os materiais didáticos para a aprendizagem que potencializam a indagação e a persuasão.

Palavras-chave: campos emocionais, dinâmica não-linear, conectividade, dinâmica de aprendizagem, aprendizagem significativa. 


\section{INTRODUCCIÓN}

El enfoque aquí presentado se centra en los procesos de interacción a nivel de alumnos al interior de la sala de clases (en la micro escala), ya que es allí donde se realiza, a través de las diversas interacciones entre personas, uno de los pilares esenciales del proceso de aprendizaje y de logros de metas del mismo: la conectividad.

Para gran parte de las actividades humanas, se construye en una primera aproximación, un "mundo" según cierto nivel de expectativas y de predectibilidad predefinidas, generándose un verdadero "mar de homogeneidad" (Capra, 1982; Connes, 1990), un reduccionismo lineal de la vida y acciones de las personas que es muy propicio y funcional a la economía y a su definición de calidad (Stiglitz, 1987; Chomsky, 2012). Pero su aplicación en enseñanza es válido en un dominio muy restringido, pues no es verdadero que más infraestructura de edificios, más computadores, más tecnologías de información y comunicación (Balanzkat, Blamire y Kefala, 2006; Marqués, 2013), más Internet (Carr, 2011), etc, provoquen, en directa proporcionalidad, más aprendizajes significativos (Rodríguez, 2010), ya que estos dependen de las formas relacionales entre las personas (Losada, 1999; Losada y Heaphy, 2004; Losada y Fredrickson, 2005; Pacheco et al., 2011). El sistema educativo es de alta complejidad (Grün y Del Caño, 2003; Laughlin, 2007; Mock, 2013), debido a que las variables fundamentales que lo caracterizan, sobre todo en el aprendizaje, están interrelacionadas entre sí, lo que es la base de todo sistema humano real.

De lo anterior, se desprende que los objetivos de este trabajo son los siguientes:

- Especificar un conjunto de variables significativas que den cuenta de las dimensionalidades que participan en un proceso de enseñanza, realizando seguimiento y registro de ellas en tiempo real.

- Validar los instrumentos de medición, según el universo muestral de estudio, por distribución estadística $\mathrm{t}$ - student e índice de Cronbach.

- Utilizar condiciones iniciales como inductor de transformación emocional.

- Aplicar métodos matemáticos de análisis a las bases de datos: Series de tiempo de Fourier, Fast Fourier Transformation (FFT) y Función de Correlación Cruzada.

- Construir las representaciones gráficas de las variables, tanto en forma bidimensional como tridimensional.

- Caracterizar las transiciones entre las dinámicas, y obtener numéricamente la conectividad, es decir, los vínculos relacionales y la transformación emocional de los alumnos en cada equipo, lo que incide directamente en la calidad de sus aprendizajes.

\section{LAS EMOCIONES}

El conjunto de emociones o estados de ánimo que posee una persona o un grupo de ellas, y que impregna las interacciones entre las mismas, junto al ambiente o entorno al cual la persona se incorpora, lo llamaremos campo emocional, a semejanza de un campo de fuerzas físico, nombre que se explica por el hecho de que las emociones poseen la capacidad de movilizar a las personas.

Las emociones juegan un papel fundamental en los aprendizajes (Maturana, 1990; Ibáñez, 2002; Pacheco, Villagran y Quiroz, 2013) que adquieren los alumnos y en las 
acciones que se emprendan para ello. Esto se desprende de lo señalado por Humberto Maturana, cuando afirma que:

Cuando hablamos de emociones son distintos dominios de acciones posibles en las personas y animales, y a las distintas disposiciones corporales que los constituyen y realizan. (...) no hay acción humana sin una emoción que la funde como tal y la haga posible como acto. (...) no es la razón lo que nos lleva a la acción sino la emoción (Maturana, 1990:20 -21).

\section{CARACTERIZACIÓN DE CADA DIMENSIÓN QUE SE MIDE EN AULA POR EQUIPO}

Se codificaron las conductas susceptibles de seguimiento observacional (Bareman y Gottman, 1989; Gottman, 1994; Baumeister et al., 2001; Losada y Heaphy 2004; Losada y Fredrickson, 2005):

Variable y, es fundamental en este trabajo y corresponde a las emociones, se ha dividido en: Positividad (POS.) cuando se manifiestan actos positivos como apoyo y comprensión en el equipo y Negatividad (NEG.) si se manifiesta en la forma de actos negativos tales como desaprobación, sarcasmo o cinismo.

Variable $\mathrm{x}$, se ha dividido en: Indagación (IND) que se relaciona con utilizar elementos didácticos (Cabero, 2001) propios de la actividad experimental y Persuasión (PER) si se emplean componentes de discusión a favor del punto de vista de alguien del equipo consiguiendo que los otros comprendan y asuman ese punto de vista.

Variable z, es constituida por Información Externa (I.E.) la que está asociada a si la información se orienta hacia fuera o desde fuera del equipo y del aula. Información Interna (I.I.): se refiere a la(s) persona(s) que abordan los temas apelando a recursos al interior de un equipo: alumno con habilidades matemáticas, otro en física, etc.

\section{METODOLOGÍA DE LA INVESTIGACIÓN}

La muestra estudiada (sin criterio de selección) es constituida por 160 alumnos (40\% mujeres y 60\% hombres) de carreras de Ingeniería en Prevención de Riesgos e Ingeniería en Procesos Industriales, de la institución de educación superior Inacap IP Santiago Sur. Estos alumnos constituyen un segmento que ingresa sólo con sus estudios secundarios terminados, sin prerrequisito de pruebas de selección universitaria, y una proporción importante de ellos conforman un grupo humano de primera generación familiar en acceder a la educación superior.

Están distribuidos en cinco cursos, de los cuales uno es de control, el de la asignatura de Mecánica, realizándoseles un seguimiento observacional. Cada curso tiene un promedio de 32 alumnos, los que se dividen, para las sesiones específicas de Laboratorio, en dos grupos de 16 alumnos, conformándose 4 equipos de trabajo con 4 alumnos cada uno, pensando en aprovechar las potencialidades del aprendizaje cooperativo y de género. La matriz de seguimiento temporal, por equipo de alumnos, está dividida en intervalos de 9 minutos (criterio de optimización atencional), hasta completar la clase de 90 minutos (Pacheco, 
Villagran y Quiroz, 2013). La emoción en la micro escala (micro emociones humanas y micro expresiones), considerando que Gottman (Bareman y Gottman, 1989) usa tiempos del orden de 1 segundo en su escala de codificación de afectos específicos de 20 categorías para estudiar las emociones posibles que puede llegar a expresar una pareja durante una conversación, escapa a los rangos de este estudio.

Cada componente de las dimensionalidades involucradas en el estudio ( $\mathrm{X}$ o Y o Z) es registrada por una persona, psicólogo o profesional capacitado. Como ilustración se muestra la Positividad que es una de las escalas de doble polaridad que componen la variable $\mathrm{Y}(t)$ (= Positividad/Negatividad. $(t))$, que es la que, posteriormente, se grafica:

\section{Positividad:}

Esquema $N^{o} 1$. Señala el dominio de doble polaridad y la escala de codificación, en su doble polaridad, de la Positividad

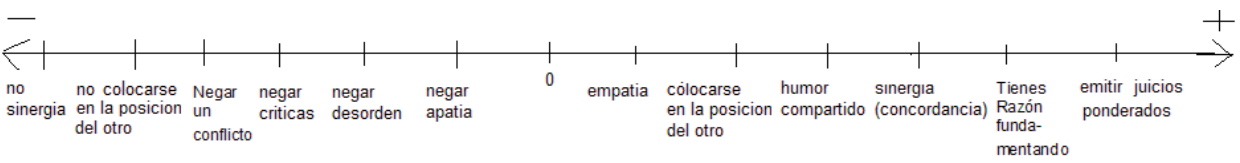

Tabla $N^{o} 1$. Presenta la conducta a seguir en el tiempo y su taxonomía de codificación.

\begin{tabular}{r|l|r|l|}
\hline & Codificación & $\mathrm{t}$ (min) & Conducta \\
\hline 6 & Emitir juicios ponderados & 0 & \\
\hline 5 & Tienes razón fundamentando & 9 & \\
\hline 4 & Sinergia & 18 & \\
\hline 3 & Humor compartido & 27 & \\
\hline 2 & Colocarse en la posición del otro & 36 & \\
\hline 1 & Empatia & 45 & \\
\hline 0 & Neutro & 54 & \\
\hline-1 & Negar apatía & 63 & \\
\hline-2 & Negar desorden & 72 & \\
\hline-3 & Negar criticas & 81 & \\
\hline-4 & Negar un conflicto & 90 & \\
\hline-5 & No colocarse en la posición del otro & & \\
\hline-6 & No sinergia & & \\
\hline total: 13 & \multicolumn{2}{|l}{} & \\
\hline
\end{tabular}

El Gráfico $\mathrm{N}^{\mathrm{o}} 1$ se produce de la Tabla de Valores para el comportamiento en el tiempo, $t$, de la variable Y (definida como cuociente relacional, para cada tiempo, entre el numeral que caracteriza la conducta registrada para la Positividad y el de aquella conducta observada para la Negatividad) (Bareman y Gottman, 1989). El primer máximo de la curva se produce al aplicar la condición inicial a los 36 minutos: 
Gráfico $N^{o} 1$. Cuociente Positividad/Negatividad versus el tiempo (caso caótico).

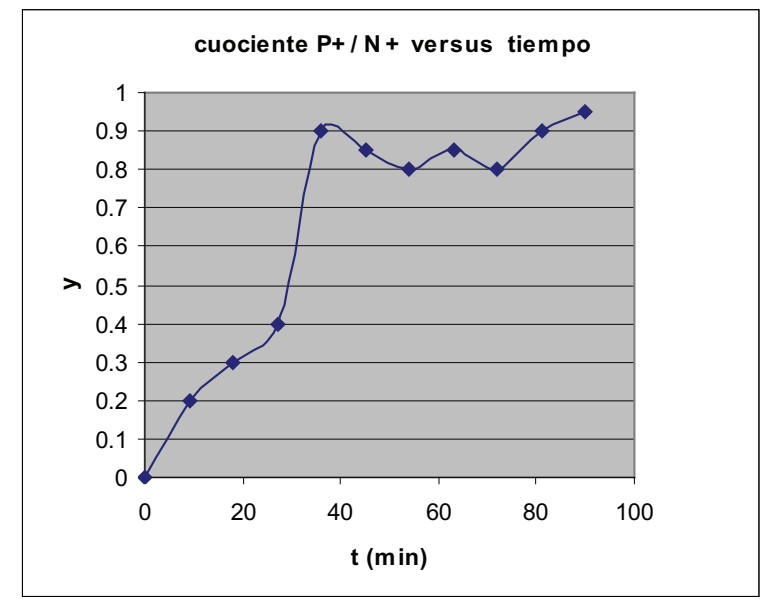

\section{CONFIABILIDAD Y EXACTITUD DE LOS INSTRUMENTOS USADOS EN LA MEDICIÓN}

El estudio de confiabilidad y exactitud se aplica a las Tablas de Valores de $\mathrm{X}(\mathrm{t}), \mathrm{Y}(\mathrm{t})$, $\mathrm{Z}(\mathrm{t})$, que son instrumentos de medición derivados o indirectos surgidos de las tablas de codificación taxonómicas. En estadística, el intervalo de confianza se corresponde con un par de números entre los cuales se estima que estará cierto valor desconocido con una determinada probabilidad de acierto. Formalmente, estos números determinan un intervalo, que se calcula a partir de los datos de una muestra, y el valor desconocido es un parámetro poblacional. La probabilidad de éxito en la estimación se representa con 1 - $\alpha$ y se denomina nivel de confianza. En estas circunstancias, $\alpha$ es llamado error aleatorio o nivel de significación, esto es, una medida de las posibilidades de fallar en la estimación a partir de tal intervalo. Para los intervalos de confianza, se aplica una distribución t - Student, pues interesa realizar comparación de medias, siendo la muestra relativamente pequeña y uniparamétrica, por cuanto depende sólo de los grados de libertad (n). El intervalo de confianza que se aplica es al nivel de $\alpha=0.05$.

La calibración de los instrumentos de medición desde el punto de vista de la exactitud se realiza a través del Test de hipótesis o Docimasia, que es una prueba de significación estadística. Se calcula, para cada dimensión del estudio, el Coeficiente de Correlación Muestral de Pearson (Canavos, 1988). Para cuantificar el nivel de fiabilidad de una escala de medida de la magnitud inobservable, construida a partir de las variables observadas, se utiliza el alfa de Cronbach (Cronbach, 1951). En psicometría, el Alfa de Cronbach es un coeficiente que sirve para medir la fiabilidad de una escala de medida.

Ilustración: cálculo del Coeficiente de Correlación Muestral de Pearson y del Coeficiente Alfa de Cronbach para la variable X = Indagación/Persuasión en el modelo de Dinámica no Lineal aplicado a las emociones y los aprendizajes.

Coeficiente de Correlación Muestral de Pearson para la dimensión fundamental, X, referida a 11ítems de las Tablas de Valores de X(t) de dos cursos diferentes: 


$$
r=\cos (\alpha)=\frac{\sum_{i=1}^{N}\left(x_{1 i}-\bar{x}_{1}\right)\left(x_{2 i}-\bar{x}_{2}\right)}{\sqrt{\sum_{i=1}^{N}\left(x_{1 i}-\bar{x}_{1}\right)^{2} \sqrt{\sum_{i=1}^{N}\left(x_{2 i}-\bar{x}_{2}\right)^{2}}}} \approx \frac{0.7368}{\sqrt{0.845 \sqrt{1.2811}}} \approx 0.7 \approx p
$$

Coeficiente Alfa de Cronbach:

$$
\begin{gathered}
\alpha_{e s t}=\frac{k p}{1+p(k-1)}=\frac{11 * 0.7}{1+0.7 *(11-1)} \approx 0.9625, \\
\mathrm{k}=\text { numero de ítems del instrumento de medición. }
\end{gathered}
$$

Intervalo de confianza al nivel de $\alpha=0.05$ :

$$
\text { Intervalo de confianza }=\bar{X} \pm t_{n-1 ; 1-\frac{\alpha}{2}} \frac{S}{\sqrt{n}}
$$

con $\bar{X}=0.55, S=0.2771, \alpha$ al nivel $0.05, r=n-1=11-1=10=$ grados de libertad donde $\mathrm{n}$ es el numero de mediciones.

$$
\begin{aligned}
& t_{n-1 ; 11-\frac{\alpha}{2}}=t_{10-1 ; 1-\frac{0.05}{2}}=t_{9 ; 0.975}=2.228(\text { Spiegel, 2000) } \\
& \text { intervalo de confianza }=0.55 \pm 2.228 * \frac{0.2771}{\sqrt{11}}= \begin{cases}0.36 & (\text { mínimo }) \\
0.74 & \text { (máximo) }\end{cases}
\end{aligned}
$$

Aplicación del Test de Hipótesis o Docimasia a la variable de Indagación/Persuasión Se desea saber si el instrumento de medición está calibrado desde el punto de vista de la exactitud.

Prueba de Hipótesis:

Se define $\mathrm{H}_{0}=$ Hipótesis nula y $\mathrm{H}_{1}=$ Hipótesis alternativa Procedimiento:

$\mathrm{H}_{0:} \mu=0.5$, el instrumento está calibrado en exactitud.

$\mathrm{H}_{1}: \mu \neq 0.5$, el instrumento no está calibrado. Hay un error sistemático.

$$
\begin{gathered}
\begin{array}{c}
t=\frac{\bar{X}-\mu}{\frac{S}{\sqrt{n}}}=\frac{0.55-0.5}{\frac{0.2771}{\sqrt{11}}} \approx 0.6, \quad \text { donde }: \\
X=0.55 ; \quad \mu=0.5 \text { equipo de control(o validamente calibrado) } \\
\quad \text { (Para realizar las mediciones y obtener } \mu \text { se ocuparon equipos } \\
\text { de seguimiento observacional diferentes) } \\
r=n-1=11-1=10
\end{array}
\end{gathered}
$$


Representación en el Gráfico $\mathrm{N}^{\circ} 2$ de la Distribución Normal de los indicadores t:

Gráfico $N^{\circ} 2$. Distribución Normal de los indicadores t.

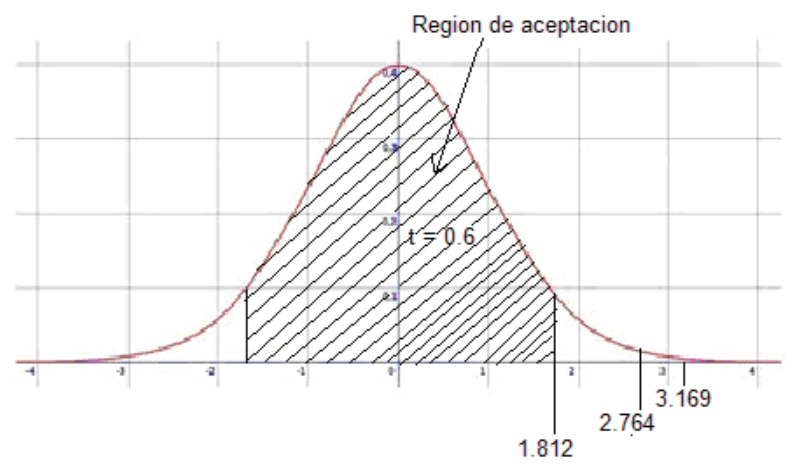

Se acepta la hipótesis de instrumento calibrado con exactitud. Igual tratamiento se realiza para las variables $\mathrm{X}$ y $\mathrm{Z}$ lo que permite, en definitiva, validar las Tablas de Valores construidas y sus respectivas representaciones gráficas.

\section{AJUSTE DE SERIES DE TIEMPO DE FOURIER A LOS DATOS EXPERIMENTALES}

Al llevar los datos a series de tiempo de Fourier (Zill, 1988), se observa la interacción entre los miembros de un equipo, detectándose procesos de mutua influencia o comportamientos entrelazados entre los componentes del equipo. Este es el ambiente del parámetro de control, $r$, esencial en el proceso de aprendizaje, referido a conductas dentro de los equipos de alumnos, que propician o obstaculizan el proceso conducente al logro de un objetivo educativo (Goleman, 2005; Varela, Thompson y Rosch, 1997). Cada serie temporal de Fourier (Zill, 1988), consecuente con el modelo matemático no lineal aplicado, tiene la forma algebraica:

$$
\begin{aligned}
\frac{d X}{d t} & =a_{0}+\sum_{i=1}^{\infty}\left[a_{i} \cos i \omega t+b_{i} \operatorname{sen} i \omega t\right] ; \quad \frac{d Y}{d t}=b_{0}+\sum_{i=1}^{\infty}\left[c_{i} \cos i \omega t+d_{i} \operatorname{seni} \omega t\right] ; \\
\frac{d Z}{d t} & =c_{0}+\sum_{i=1}^{\infty}\left[e_{i} \cos i \omega t+f_{i} \operatorname{seni\omega t}\right]
\end{aligned}
$$

$\mathrm{Al}$ adaptar una serie de tiempo continuo de Fourier a una gráfica experimental de la forma $Y(t)$ versus $t, d Y(t) / d t$ versus $t$ (de igual manera para $X(t)$ versus $t, Z(t)$ versus $t, d X$ $(\mathrm{t}) / \mathrm{dt}$ versus $\mathrm{t}$ y dZ (t)/dt versus $\mathrm{t})$, se obtienen los gráficos: 
Gráfico $N^{o} 3$. La línea negra es la variación en el tiempo de la dimensión Y(= Positividad/ Negatividad). La otra es la curva de ajuste según Fourier. En el gráfico derecho la línea oscura es la razón de cambio de $\mathrm{Y}$ en el tiempo, t.
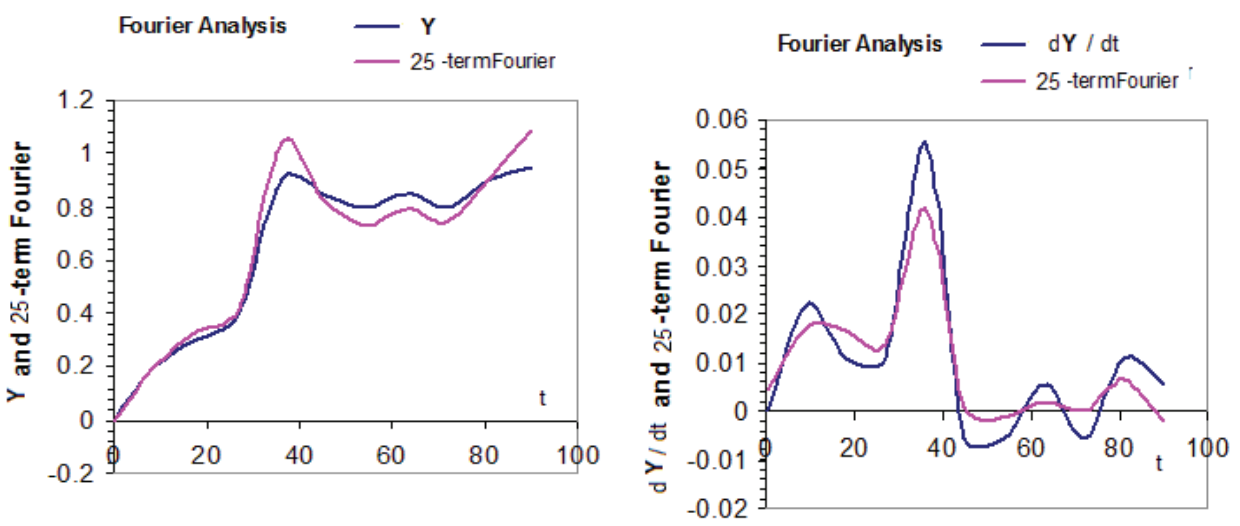

\section{CONDICIONES INICIALES CONTEXTUALIZADAS}

Al aplicar las condiciones iniciales (Zill 1988; Faires y Burden, 2004), percibida la homogeneidad del campo emocional inicial del curso (que puede contener conductas de estancamiento, indiferencia, ansiedad, etc.), se activa una nueva disposición emocional que impulsa al equipo hacia los objetivos presentados. El tipo de condición inicial dependerá del nivel de complejidad de un contenido, de cuán factible sea contextualizarlo, del carácter de la dificultad que tenga el alumno (Beilock et al., 2010), etc., lo que le da un carácter de alta complejidad (Schuster 1995; Sprott, 2006).

Se usó el criterio de aplicar la condición inicial a los 36 minutos de comenzada la sesión lectiva de 90 minutos ya que, en general, los alumnos no han leído o bien comprendido sus Guías de Trabajo o de sesión Experimental. El profesor debe invertir tiempo en introducirlos a la actividad del día (tiempo que importa para el logro de los fines). Las dificultades que surgen del trabajo de cada equipo, junto con la percepción del ambiente de campo emocional inicial, las materias a desarrollar y las directrices valóricas a transmitir, son las que disponen el tipo de condición inicial, de ahí la necesidad de contextualizarlas. Al romper la simetría del campo emocional inicial, se genera el impulso y esfuerzo necesario hacia la meta.

En esta investigación, se pudo comprobar, del seguimiento observacional de las actividades de los equipos de alumnos, la efectividad de las condiciones iniciales específicas tales como: experimento demostrativo acotado a una fracción de tiempo respecto del período lectivo de la clase; sesión de laboratorio planificada dentro de la asignatura; asignatura de laboratorio independiente, pero que sea la contraparte práctica de la asignatura teórica, con la que entrecruza contenidos optimizando y mejorando los objetivos de aprendizaje; prácticas breves asociadas al perfil profesional que se pretende para el estudiante; video corto (10 o 15 minutos), estructurado en una narrativa simple y accesible que se aboque valóricamente a temáticas específicas de los contenidos; presentación breve de algún tema específico del programa de asignatura, por un investigador docente de personalidad carismática y empática (testimonio), etc. 


\section{ANÁLISIS DE DATOS Y GRÁFICOS}

De las gráficas construidas, y basado en el dominio de conductas susceptibles de verificación experimental (o de seguimiento experimental) propuestos en los dominios de definición para X, Y, Z (Gottman 1994; Losada y Heaphy 2004; Losada y Fredrickson 2005), se desprende:

$1^{\circ}$. Se logran los objetivos de aprendizaje si se manifiestan equilibradamente las variables de indagación/persuasión, es decir, de las conductas características de indagación (13, 6 de campo positivo y 6 de campo negativo, más una neutra) y de persuasión (13, 6 de campo negativo y 6 de campo positivo, más una neutra), y no debiera dominar una sobre la otra. Lo notable del trabajo experimental en un laboratorio de física es la concatenación gradual de las conductas referidas a una medición y asociadas a la indagación, no se da una segunda conducta si no se han cumplido las anteriores. Luego, es fundamental que las actividades relacionadas a cada conducta contenida en la persuasión, estimulen las de indagación. Las gráficas muestran que el dominio de la persuasión en sesiones de trabajo experimental conlleva, necesariamente, a una disipación de los esfuerzos, generándose un reducción en amplitud de la forma $e^{-k f(t)}$ que arrastra a las otras variables $(\mathrm{Y}, Z)$.

Gráfico $N^{o} 4$. Cada curva representa el comportamiento en el tiempo de la variable X (definida como cuociente Indagación/persuasión) para cada dinámica: Atractor débil $\left(\mathrm{X}_{d}\right)$, fijo $\left(\mathrm{X}_{f}\right)$ y caótico $\left(\mathrm{X}_{c}\right)$.

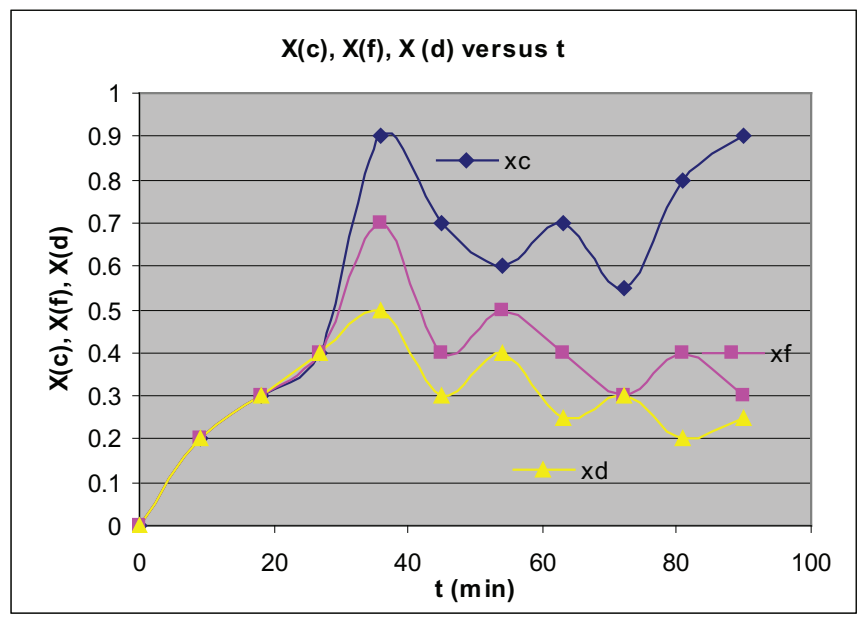

Al realizar el seguimiento de $\mathrm{X}(t)$ para las distintas dinámicas, surge de sus gráficos un efecto "resonante" para los equipos que logran los objetivos de aprendizaje y que originan dinámicas del tipo atractor caótico. Este efecto funcional evolvente sobre $\mathrm{X}(t)$ es bien descrito por formas exponenciales positivas de la forma $e^{\lambda g(t)}$ y determina un incremento en el tiempo de $\mathrm{X}(t)$. Es decir, surge el auto refuerzo o autoestímulo inter-alumnos, los componentes del equipo apoyándose en la generación del campo emocional Y, positivo, con elementos tales como el humor, la empatía, la autogeneración de un clima de confianza, etc. 
$2^{\circ}$. Si bien la variable $Y$, de las emociones, que involucra la proporción entre positividad y negatividad, junto a las conductas asociadas a numerador y denominador, es la que impulsa el proceso, si no es adecuadamente canalizada en la conectividad inter-alumnos del equipo también tiende a atenuarse disipándose con un decaimiento semejante al de una exponencial negativa en función del tiempo, $e^{-\theta \mathrm{h}(t)}$.

Gráfico $N^{\circ} 5$. Cada curva representa el comportamiento en el tiempo de la variable Y (definida como cuociente Positividad/Negatividad) para cada dinámica:

Atractor débil $\left(\mathrm{Y}_{d}\right)$, fijo $\left(\mathrm{Y}_{f}\right)$ y caótico $\left(\mathrm{Y}_{c}\right)$.

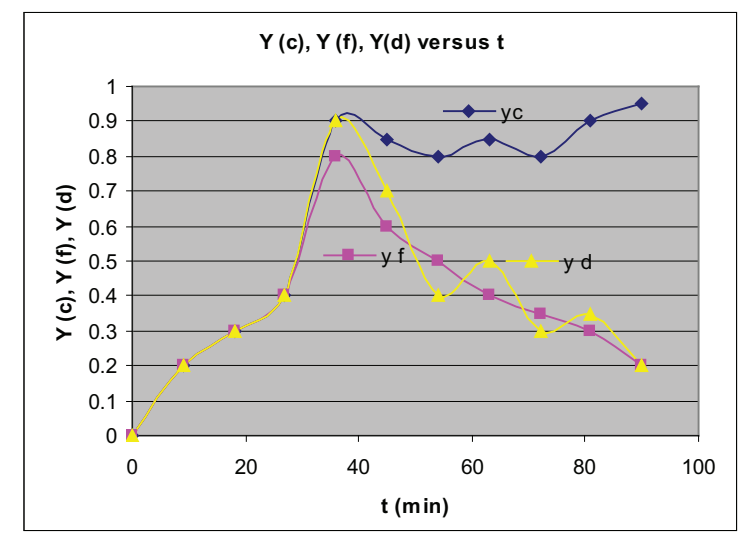

Al generar la condición inicial un ambiente de campo emocional nuevo, éste aporta el impulso y la energía hacia el objetivo. Se observa de las gráficas que los equipos de alto rendimiento son capaces de administrar en el largo plazo esta energía "emocional". No así los equipos de rendimiento medio y bajo que, a pesar de haber alcanzado un "estado excitado" y de energía asociada, semejante al caso de alto rendimiento, no lo "disponen" bien en el tiempo, difundiéndose inter y extra-equipo, afectando seriamente el logro de las metas (desorden, dispersión del trabajo, bromas pesadas, poco compromiso con la rigurosidad de las mediciones, etc.).

$3^{\circ}$. Información interna/Información externa. Esta variable tiene en los equipos de trabajo de laboratorio en física una característica singular. De lo observado en los diferentes equipos de alumnos que exhiben más compromiso con los objetivos propuestos, éstos están orientados hacia el trabajo al interior del equipo (Información interna) y a la búsqueda de respuestas dentro de él. Pero esto no es una característica general en los equipos estudiados, lo que se ve reflejado en los resultados gráficos finales. El dato que más se registra en el seguimiento observacional, es el de la orientación externa, es decir el buscar respuestas fuera del equipo, en particular en el profesor. Si bien esta afirmación es especulativa, quizás esto tenga que ver con una deformación educacional que tiende más a la imitación o repetición (o dicho groseramente, a "copiar" el trabajo de los otros equipos o seguir pautas de la autoridad) en el contexto en que se realizan las observaciones. Luego, el factor dominante en la razón Información interna/Información externa es el denominador, predominando las conductas para él definidas. Es así que los guarismos son inferiores a 
1, en general (ausencia de equilibrio). Esto determina un efecto de amortiguamiento sobre todas las dinámicas que surgen de los datos experimentales.

Gráfico $N^{\circ} 6$. Cada curva representa el comportamiento en el tiempo de la variable $z$ (definida como cuociente Información Interna/Información Externa) para cada dinámica: Atractor débil $\left(\mathrm{Z}_{d}\right)$, fijo $\left(\mathrm{Z}_{f}\right)$ y caótico $\left(\mathrm{Z}_{c}\right)$.

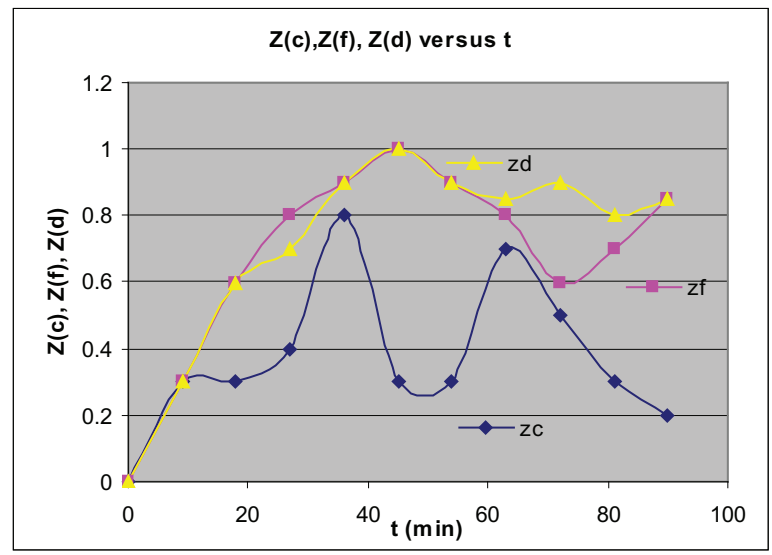

El docente debe leer, previamente, la "hoja de vida" (emocional y de rendimiento) de cada alumno con el propósito de seleccionar con rigurosidad la condición inicial contextualizada adecuada, tarea que cualquier docente experimentado puede implementar. Dado que el seguimiento observacional es durante un semestre lectivo, es conveniente modificar la planificación de contenidos del programa de asignatura, dándole una estructura modular de 12 módulos (pueden ser menos o más): Cada módulo es asumido por cada equipo como un emprendimiento o empresa educativa. Los módulos se organizan con objetivo, introducción, materiales, procedimiento, Tablas de valores (si compete), gráficos (si compete), análisis (conceptual-matemático, fortalezas- debilidades-costos (si compete)), conclusión y bibliografía (literatura, Internet, Web). Evaluándose, cada módulo por alumno y equipo, según las modalidades de: informe, interrogación oral (se selecciona un alumno, del equipo, al azar), interrogación escrita, disertación (del equipo).

Al final de cada módulo, se presentan al equipo los resultados de las dimensiones medidas y se les explica la forma en que éstas inciden en el logro de los objetivos. Esto les permite corregir sus dinámicas de trabajo en los módulos posteriores (el fracaso también es aprendizaje). La influencia de la condición inicial contextualizada y de la variable Y, de las emociones, en el sentido de contener y propiciar una Positividad/Negatividad equilibrada y sincera al interior del equipo, es fundamental, por las implicancias valóricas respecto del trabajo bien hecho: compromiso, esfuerzo y honestidad. Al final del semestre, se analiza el historial completo que es el que determina el éxito, el éxito relativo o fracaso (en el peor de los casos) de la empresa o emprendimiento educativo del equipo, por módulo y según los contenidos. El proceso es simple de implementar, pero requiere de las destrezas de los observadores, en particular neutralidad (en una investigación posterior se pueden usar, además, cámaras de video). 


\section{REPRESENTACION GRÁFICA DE LAS DISTINTAS DINÁMICAS}

De las gráficas se desprende que los equipos que logran los objetivos propuestos, alto rendimiento, exhiben una relación equilibrada entre indagación/persuasión. Sin embargo, para la razón positividad/negatividad hay una mayor influencia del numerador. Si fuera muy dominante la positividad los alumnos de los equipos, éstos se vuelven demasiados condescendientes entre ellos, lo que tampoco propicia el logro de las metas (Gottman, en su estudio de relaciones perdurables para matrimonios, propone sobre 5.8 como adecuados (Gottman, 1994)).

Al estudiar la razón Información interna/Información externa, se obtiene que su valor numérico es del orden del 60\%, o menos. La observación del desempeño de los equipos de alumnos en sesiones de tipo experimental, para el cuociente indicado, refleja lo que ocurre. La mayoría de los equipos muestran un fuerte predominio de Información externa, aparecen "volcados" hacia el exterior del equipo y buscan respuestas fuera del equipo. Pero los buenos equipos de alumnos (para trabajar en experimentos), al revés de lo observado en la muestra en estudio, muestran una orientación hacia la búsqueda de Información interna (apelando a las experticias de los mismos integrantes del equipo), a esto ayuda la concentración que requiere el trabajo en equipo en un laboratorio.

El comportamiento así descrito de las variables, determina una dinámica característica y que se denomina caótica, según muestra la figura (Kaspar y Schuster, 1986; Sprott, 2006):

Gráfico $N^{\circ} 7$. La gráfica es la representación tridimensional de las columnas de X, Y, Z -cada una de 1.000 datos -, generadas en el seguimiento en el tiempo de los diferentes equipos de alumnos que satisfacen la dinámica de atractor caótico. Aproximada,

la geometría posicional del centroide (región circular) (Losada, 1999).

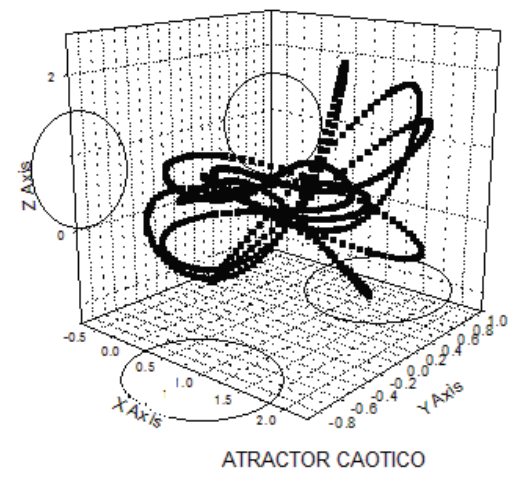

A diferencia del caso presentado antes, los equipos de rendimiento medio muestran valores numéricos decrecientes en el tiempo para indagación/persuasión (X) y positividad/ negatividad (Y). Esto significa que, en el caso de la variable $\mathrm{X}$, el denominador es dominante, incurriendo los equipos en conductas de tipo persuasivo, cuando en realidad lo que se requiere en los procesos de medición de laboratorio es capacidad indagatoria de los fenómenos con registro de datos con instrumentos de medida. Se genera, al interior 
de los equipos, un campo emocional con dominio de negatividad y que se explicita a través de apatía, desorden, negación de conflictos, etc. No así para la Información interna/ Información externa, que muestra una clara tendencia a buscar respuestas ya sea fuera o dentro del equipo.

La gráfica que se presenta a continuación, nos muestra la forma cómo las variables X, Y, Z se combinan para dar origen a la dinámica de punto fijo (Kaspar y Schuster, 1986; Sprott, 2006):

Gráfico $N^{\circ} 8$. La gráfica es la representación tridimensional de las columnas de X, Y, Z -cada una de 1.000 datos-, generadas en el seguimiento en el tiempo de los diferentes equipos de alumnos que satisfacen la dinámica de atractor de punto fijo. En forma aproximada, la geometría de posición del centroide (región circular)(Losada, 1999).

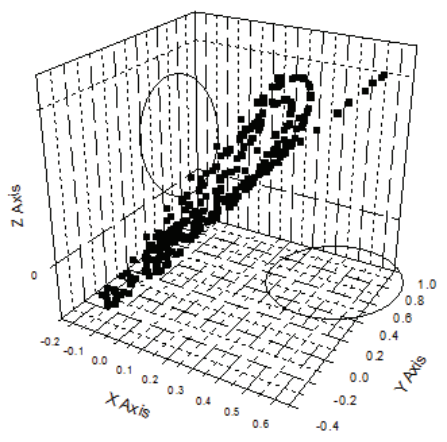

Si los equipos exhiben un rendimiento bajo, los valores numéricos para indagación/ persuasión (X) y positividad/negatividad (Y) son bastante inferiores a la unidad y marcadamente decrecientes, mostrando los equipos de trabajo experimental una muy fuerte tendencia hacia la persuasión y la negatividad. Al igual que en el caso anterior, respecto a la Información interna/Información externa, los equipos de alumnos que buscan soluciones despliegan igual número de conductas, tanto para el denominador como para el numerador, pero sin un procedimiento definido, es decir limitándose a buscar respuestas sin análisis de validación de las mismas. El aprendizaje se reduce a niveles mínimos, pues a través de la copia de resultados a otros equipos o improvisando una solución, es muy difícil que como equipo (y según el aporte personal de sus componentes) se produzca un aprendizaje significativo. La gráfica que se presenta a continuación nos muestra la forma cómo las variables $\mathrm{X}, \mathrm{Y}, \mathrm{Z}$ se combinan para dar origen a la dinámica de punto débil (Kaspar y Schuster, 1986; Sprott, 2006): 
Gráfico $N^{\circ} 9$. La gráfica es la representación tridimensional de las columnas de X, Y, Z -cada una de 1.000 datos-, generadas en el seguimiento en el tiempo de los diferentes equipos de alumnos que satisfacen la dinámica de atractor de punto fijo débil. Aproximada, la geometría posicional del centroide (región circular) (Losada, 1999).

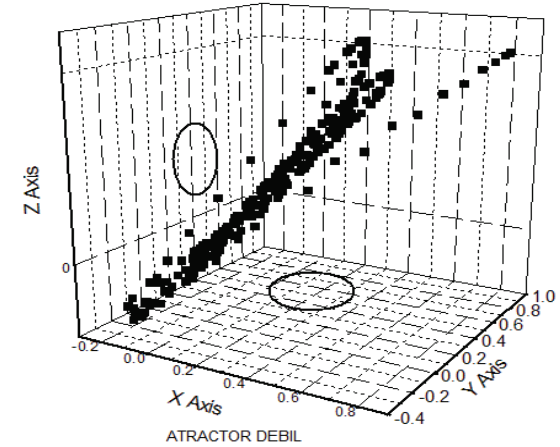

La proyección de las curvas tridimensionales - según cada plano XY, XZ, YZpermite obtener en forma gráfica los centroides asociados a los focos de los atractores que mantienen las trayectorias dentro de límites (Losada, 1999; Losada y Heaphy, 2004). Los valores numéricos indican la influencia que cada variable ejerce sobre la otra. Esta visión macroscópica de las dinámicas de interacción no lineales señala la forma en que se manifiestan los vínculos relacionales, o conectividad (r), entre personas del equipo.

\section{SERIES DE TIEMPO DE FOURIER Y MEDIAS MÓVILES}

Usando Series de tiempo y medias móviles respecto de las Tablas de Valores para Y, se obtiene para cada dinámica un valor promedio de convergencia del cuociente Positividad/ Negatividad, P/N, parámetro que permite caracterizar y clasificar las dinámicas de trabajo:

$$
\left\langle\left(\frac{P}{N}\right)\right\rangle=\left\{\rightarrow 0.375_{\text {DEBIL }}, \quad 1.95_{\text {MEDIO }}, \quad 5.25_{\text {CAOTICO }}\right\}
$$

Al emplearlos como referencia en esta primera aproximación, estos valores están en concordancia con las investigaciones realizadas por Gottman respecto a las relaciones de matrimonio (Gottman, 1994), y de Losada para el rendimiento de equipos de personas en actividades de tipo económico (Losada y Heaphy, 2004).

\section{LA FUNCIÓN DE CORRELACIÓN CRUZADA}

Dadas dos funciones $f(t)$ y $g(t)$, su función de correlación cruzada (Sprott, 2006) está dada por:

$$
R_{\mathrm{fg}}(\tau)=\mathrm{f}(\mathrm{t}) * \mathrm{~g}(-\mathrm{t})=\int_{-\infty}^{\infty} \mathrm{f}(\mathrm{t}) \mathrm{g}(\tau+\mathrm{t}) \mathrm{dt}
$$


La función de correlación cruzada se puede interpretar como la medida de la coincidencia entre dos imágenes, para varias cantidades de corrimiento. Es posible medir los nexos por medio de la función de correlación cruzada discretizada aplicada a todos los datos de series de tiempo que se generaron del trabajo, en cada equipo, para X, Y, Z. Esta función proporciona una medida de la fuerza y de cuánto demora la conducta particular de una persona, en el tiempo, en enlazar con la conducta de otra persona. El cálculo de la influencia del ambiente emocional del equipo en la Indagación y Persuasión da, comparativamente, para $\mathrm{C}_{\mathrm{XY}}$ :

$$
\left\{0.13_{\text {DÉBIL }}<0.16_{\text {MEDIO }}<0.44_{\text {CAÓTICO }}\right\}
$$

Esto comprueba en forma matemática la hipótesis de trabajo: si los equipos propician en su interior relaciones con emociones positivas (alta conectividad entre los componentes del equipo), esto favorece el logro de las metas educativas. Si para cada dinámica se grafica $\mathrm{C}_{\mathrm{XY}}$ en el tiempo y se determina el máximo, el producto de ese valor por el tiempo de ocurrencia del máximo da la conectividad, $\mathrm{r}$, por caso: $\mathrm{r}_{\text {САотіса }}=0.81 * 36=29.16 \sim 29, \mathrm{r}$ MEDIO $=0.56 * 36=20.16 \sim 20, \mathrm{r}_{\text {DEBIL }}=0.45 * 36=16.2 \sim 16$, respectivamente.

Dado que $C x y$ representa la influencia que ejercen entre sí las variables de Indagación/ Persuasión con la de Positividad/Negatividad, en el caso caótico "bordea" el 50 \%, mientras que en los dos casos restantes, es inferior al $20 \%$. De la misma manera, se observa que en los grupos de rendimiento medio y bajo se potencia la influencia de la negatividad en la razón Positividad/Negatividad y en la proporción Orientación interna/Orientación externa, esto es, una forma de equilibrio que muestra una tendencia a obtener soluciones sin importar los medios.

\section{GRÁFICO DE LA CONECTIVIDAD VERSUS RENDIMIENTO}

La conectividad puede ser definida como la capacidad que muestran los miembros del equipo para expandir con sus acciones las acciones de los demás, y para expandir sus propias acciones a partir de las acciones de los otros (Cacioppo y Berntson, 1999; Echeverria, 2005).

Así, en particular para la relación-influencia de Y sobre X, según las diferentes dinámicas: el Gráfico $\mathrm{N}^{\mathrm{0}} 10$ da la Conectividad por cálculo del centroide, correlación cruzada (Pacheco, Villagran, y Quiroz, 2013), por el método recursivo teórico según MatLab (Nakamura, 1997) del modelo no lineal aplicado (Lorenz,1976; Grassberger y Procaccia, 1982; Kaspar y Schuster, 1986; Pacheco, Villagran y Guzmán, 2015) y valor promediado: 
Gráfico $N^{o} 10$. Tabla de Valores y Gráfico de la Conectividad versus niveles de rendimiento académico, entendido esto último como aprendizajes significativo.

\begin{tabular}{|l|c|c|c|c|}
\hline & \multicolumn{3}{|c|}{ CONECTIVIDAD } & \\
\hline RENDIMIENTO & TEÓRICA & CENTROIDE & CORRELACION & PROMEDIO \\
\hline BAJO & 16.5 & 20.15 & 16 & 17.5 \\
\hline MEDIO & 19.5 & 22.1 & 20 & 20.5 \\
\hline ALTO & 31 & 26 & 29 & 28.7 \\
\hline
\end{tabular}

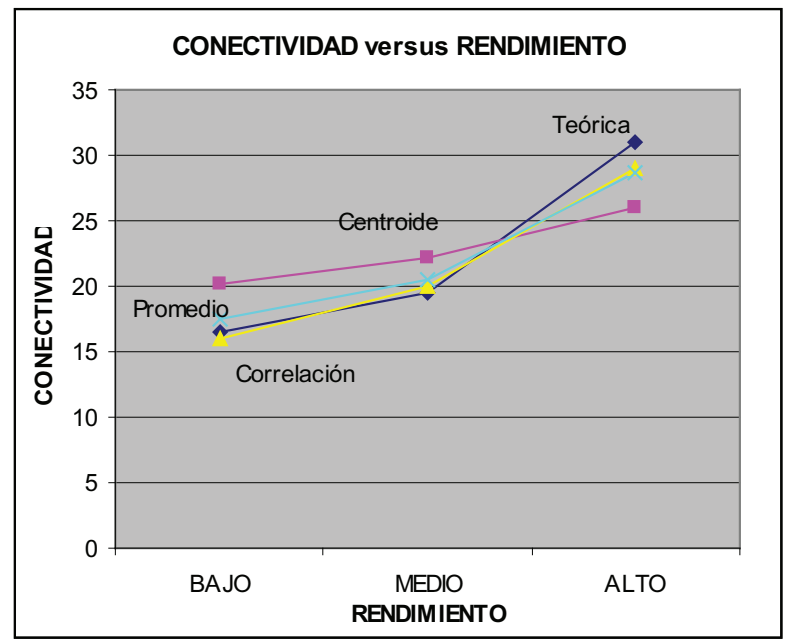

Del seguimiento observacional (Bareman y Gottman, 1989) de las dimensionalidades del modelo se extrae la información del trabajo de los equipos de alumnos (Treisman, 1992; Beilock et al., 2010). Al confirmarse la hipótesis central de este estudio se puede afirmar, con alta correlación, que al estar mediatizadas por el cuociente Positividad/Negatividad, la variable Y de las emociones, su influencia e interdependencia en formas no lineales incide directamente sobre el rendimiento de los equipos de trabajo.

\section{RESULTADOS Y ANÁLISIS}

Como corolario de los procedimientos aplicados, se presenta el resultado del uso de la condición inicial contextualizada, el rendimiento, el logro de aprendizaje significativo y el cuociente Positividad/Negatividad, respecto a una actividad (de un total de 10) de Laboratorio de Física, denominada Teoría del Error. Se señala, además, los porcentajes totales de equipos, por curso, cuya administración de su proceso de aprendizaje es:

(a) sin condición inicial contextualizada (S.C.I.C.), y

(b) con condición inicial contextualizada (C.I.C.), basada en campos emocionales y ejes valóricos, 
Actividad: Teoría del Error

Objetivo: Determinar la densidad, con su error, de una pieza cilíndrica de acero.

Se considera el aprendizaje significativo en la actividad de Laboratorio si el equipo obtiene, luego de aplicar los procedimientos indicados en la Guía de Laboratorio respectiva, la densidad del acero.

La tarea para el profesor indica que debe realizar la introducción y aplicar la Condición Inicial Contextualizada (C.I.C.) en 4 cursos, dejando el quinto curso en calidad de control Sin Condición Inicial Contextualizada (S.C.I.C.) (los cinco cursos en horarios diferentes)

El procedimiento para el alumno contiene la secuencia de etapas: definir geometría del cuerpo y variables a medir; conocer y calibrar los instrumentos de medida; medir y emplear unidades de medida; registro de mediciones en Tablas de Valores; utilizar herramientas estadísticas (calcular media y desviación estándar); aplicar propagación de errores.

Calcular la densidad con su error y comparar el valor con el de la densidad extraída de Tablas (por ejemplo: ver Serway, 1997)

Esta secuencia determina que el procedimiento conduzca a las posibilidades:

1. Procedimiento incorrecto / Densidad incorrecta

2. Procedimiento correcto / Densidad incorrecta

3. Procedimiento correcto / Densidad correcta

que están en directa relación con el cuociente Positividad/Negatividad de las emociones $\mathrm{y}$ que se traduce en Bajo rendimiento $=\mathrm{B} . \mathrm{R} .=0.375$, Medio Rendimiento $=$ M.R. $=1.95$ y Alto Rendimiento $=$ A.R. $=5.25$, según muestran las tablas comparativas finales del proceso:

\begin{tabular}{|c|c|c|}
\hline PROCEDIMIENTO & CONECTIVIDAD, $\mathrm{r}$ & Pos./ Neg. \\
\hline 1. B.R. & 20 & 0.375 \\
\hline 2. M.R & 22 & 1.95 \\
\hline 3. A.R. & 26 & 5.25 \\
\hline
\end{tabular}

\begin{tabular}{|l|c|c|c|c|c|c|c|c|c|}
\hline \multicolumn{1}{|c|}{ EQUIPOS POR CURSO } & 1 & 2 & 3 & 4 & 5 & 6 & 7 & 8 & $\%$ \\
\hline CURSO DE CONTROL (S.C.I.C.) & AR & MR & MR & BR & BR & BR & & & 16.66 \\
\hline CURSO 1 (C.I.C.) & AR & AR & AR & AR & MR & BR & & & 66.66 \\
\hline CURSO 2 (C.I.C.) & AR & AR & MR & AR & BR & MR & AR & AR & 62.5 \\
\hline CURSO 3 (C.I.C.) & AR & AR & AR & MR & AR & BR & AR & & 71.43 \\
\hline CURSO 4 (C.I.C.) & AR & AR & MR & AR & AR & BR & AR & & 57.14 \\
\hline
\end{tabular}


Lo que confirma la influencia de la condición inicial contextualizada y de las emociones en el proceso.

Se desprende de esta investigación, en forma preliminar, que las variables que ejercen una alta influencia en la calidad del aprendizaje son conductas observables asociadas a las emociones de las personas (factor humano), no los recursos materiales, subordinándose el logro del objetivo a si estos factores humanos se manifiestan en los equipos de trabajo de alumnos o no. Esta característica fundamental permite definir la calidad en el aprendizaje como: aquella en la cual la aplicación de las condiciones iniciales influencian los campos emocionales, los que a través de la razón Positividad/Negatividad se interrelacionan con la Indagación/Persuasión e Información Interna/Información Externa, fomentando o restringiendo la generación, el crecimiento y el fortalecimiento de la conectividad inter-personas de un equipo de alumnos, lo que permite u obstaculiza el logro de aprendizajes significativos. Esta definición quita prioridad a la fijación por los resultados, una de las grandes debilidades de la enseñanza, enfatizando los procedimientos y conectividades en los equipos para alcanzar un objetivo educativo. Puesto que el proceso de seguimiento de las actividades de los equipos se basa en conductas observables, ello permite su modificación por el propio convencimiento del equipo (lo que forma parte del aprendizaje) o, en última instancia (lo que debe evitarse y emplearse sólo en casos extremos), por intervención del profesor.

\section{CONCLUSIONES}

Respecto a los conceptos estadísticos aplicados a los instrumentos de medida y a las técnicas procedimentales, se concluye que éstos se encuentran en rangos de confiabilidad y validación adecuados. El procedimiento aplicado da una visión numérica cuantitativa que permite conclusiones cualitativas muy acertadas respecto del funcionamiento de los equipos observados y de la forma en que las emociones son determinantes en el logro de los objetivos de aprendizaje.

El uso de las escalas de tiempo graduadas a intervalos de 9 minutos es adecuado, en una primera aproximación, pues el énfasis está en cómo el proceso conduce al objetivo y en la forma en que la evolución de las variables observadas (X, Y, Z) llevan a un aprendizaje significativo, proceso que no es instantáneo. Es poco probable que exista una comprensión de conceptos o instrumentos referidos a la física en 1 segundo.

Al construir los gráficos en el espacio de fase de X, Y, Z, se proyectan en los distintos planos la "nube" de puntos. Sus centroides entregan, numéricamente, la conectividad, es decir, la influencia y relación que ejerce cada variable sobre la otra, confirmándose su naturaleza no lineal. Esto se verifica, además, a partir de las series de tiempo de Fourier y de la función de correlación cruzada, que es la que se presenta en este trabajo. La transición entre las dinámicas débil, media y caótica también es dada por el parámetro $r$, la conectividad, siendo sus valores experimentales para cada caso, 16, 20 y 29 respectivamente. Estos valores numéricos son un indicador del aprendizaje significativo de los equipos, revelándose, en el orden dado, si es bajo, medio o alto. Los valores numéricos confirman y fundamentan que quien impulsa la transformación y la transición entre dinámicas es la evolución del campo emocional.

Se requiere mayor rigor en la comprensión de la calidad (Álvarez-Tostado, 1997).) en su relación con los aprendizajes significativos, pues aparece ligada al cuociente Positividad/ 
Negatividad y, según lo señalan las series de tiempo, los valores de convergencia están en números bien definidos -0.375 (débil), 1.95 (medio), 5.25 (caótico)-, indicando cuál es el factor dominante de la fracción. Esto permite construir un dominio de validez para la calidad del proceso educativo, lo que incide directamente en la percepción, inserción y continuidad de los alumnos en sus aprendizajes, así como la valoración que hacen de todo ello. Este aspecto es crucial en este estudio, desde el punto de vista de evitar la deserción o abandono definitivo de las diversas profesiones que estudian los jóvenes (Pacheco, Villagran y Quiroz, 2013).

En su trabajo en equipo, los alumnos aprenden en el hacer el trabajo colaborativo. Más aún, aquello se hace en función de un "gran" objetivo: el descubrimiento de sí mismo, de los demás y de la naturaleza, comprendiendo que estos procesos imponen sacrificios de tipo emocional y deponer actitudes e intereses personales en beneficio de los componentes del equipo y del equipo mismo. En el segmento de alumnos analizado, muchos están imbuidos de una dinámica emocional que, las más de las veces, se alimenta de cierta negatividad y fatalismo (Otten y Jonas, 2014). Aún así, su actitud es claramente aspiracional, en el sentido de proponerse metas que los ayuden a salir de este verdadero "pozo" de potencial emocional.

Se ha creado, en suma, un procedimiento riguroso que abarca las siguientes etapas: observación, seguimiento y registro experimental, uso de condiciones iniciales, graficación, modelización teórica, determinación de parámetros significativos del estado de evolución, clasificación de calidad y rendimientos; lo que, desde la perspectiva de la evolución de los campos emocionales, permite optimizar el aprendizaje.

\section{REFERENCIAS BIBLIOGRÁFICAS}

Álvarez-Tostado, C. (1997). Calidad de la Educación. Entre el eslogan y la utopía. Buenos Aires: Magisterio del Río de la Plata.

Balanzkat, A., Blamire, R. y Kefala, S. (2006). The ICT Impact Report: A Review of Studies of ICT Impact on Schools in Europe. Recuperado el 11 de noviembre de 2012, de: http://insight.eun. org/ww/en/pub/insight/misc/specialreports/impact_study.htm.

Bareman, R. y Gottman, J.M. (1989). Observación de la Interacción: introducción al análisis secuencial. Madrid: Morata

Baumeister, R., Bratslavsky, E., Finkenauer, C. y Vohs, K. (2001). Bad is stronger than good. Review of General Psychology, Vol. 5, No. 4, 323-370.

Beilock, S.L., Gunderson, E.A., Ramirez, G. y Levine, S.C.(2010). Female teachers' math anxiety impacts girls' math achievement. Proceedings of the National Academy of Sciences, 107 (5), 1860-1863.

Cabero, J. (2001).Tecnología Educativa, Diseño y Utilización de Medios para la Enseñanza. Madrid: Paidós

Capra, F. (1982). El punto Crucial. Buenos Aires: Estaciones

Canavos, G.C. (1988). Probabilidad y estadística. Mexico D.F.: McGraW-Hill

Carr, N. (2011). ¿Qué está haciendo Internet con nuestras mentes? Superficiales. México D.F.: Taurus

Chomsky, N. (2012). Cómo funciona el mundo. Conversaciones con David Barsamian. Buenos Aires: Katz Editores

Connes, A. (1990). Noncommutative Geometry. San Diego: Academia Press 
Cronbach, L. J. (1951). Coefficient alpha and the internal structure of tests. Psychometrika, 16 (3), 297-334.

Faires, J. D. y Burden, R. (2004). Métodos Numéricos. Madrid: Brooks / Cole, Division of Thomson Learning, Inc.

Goleman, D. (2005). La Inteligencia Emocional. Barcelona: Cairos

Gottman, J. (1994). What predicts divorce?. The relationship between marital processes and marital outcomes. New Jersey: Lawrence Erlbaum Associates

Grassberger, P. y Procaccia, I. (1982). Characterization of Strange Attractors. Physical Review Letters, 50, 346-349.

Grün, E. y Del Caño, E. (2003). Ensayos sobre Sistémica y Cibernética. Buenos Aires: Dunken

Ibáñez, N.(2002). Las Emociones en el Aula. Estudios Pedagógicos, nº 28, 31-45.

Kaspar, F. y Schuster, H.G. (1986). Easily calculable measure for the complexity of spatiotemporal patterns. Physical Review, A 36, 842-848.

Marqués, P. (2013). ¿Por qué no mejoran las notas de los alumnos con las TIC?. Recuperado el 20 de agosto de 2013 de: http://peremarques.blogspot.com/

Maturana, H. (2001). Emociones y Lenguaje en Educación y Política. Santiago de Chile: Dolmen

Mock, T. (2013). Climate Change Hill upset vital ocean chemical cycles. Journal Nature Climate Change. Londres: University of East Anglia, press release 64 / 13

Monzó, J. (2008). Pensamiento Sistémico. Teoría del caos: una mariposa en el reloj. Recuperado el 7 de octubre 2012 de: http://www.blogger.com/profile/16346186836123661127.

Nakamura, S. (1997). Análisis Numérico y Visualización Gráfica con Matlab. México D.F.: Pearson

Laughlin, R. B. (2007). Un universo diferente. La reinvención de la física en la edad de la emergencia. Buenos Aires: Katz

Lorenz, E. (1976). Deterministic nonperiodic flow. Journal of Atmospheric Sciences, 20, 69.

Losada, M. (1999). The complex dynamics of high performance teams. Mathematical and Computer Modelling, 30(9-10),179-192.

Losada, M. y Fredrickson B.L.(2005). Positive Affect and Complex Dynamics of Human Flourishing. American Psychologist, 60(7), 678-686.

Losada, M. y Heaphy, E. (2004). The role of positivity and connectivity in the Performance of Business Teams. American Behavioral Scientist, Vol. 47, Nº, 740-765.

Otten, M., Jonas, K.(2014). Humiliation as an intense emotional experience: Evidence from the electro-encephalogram. Social Neuroscience, Volume 9, Issue 1, 23-35.

Pacheco, P., Villagrán, S., Guzmán, C., Muñoz, I., Quiroz, E., Vásquez, L., Donoso, R. (2011). Modelo de Dinámica de sistemas complejos para analizar las emociones, las formas relacionales y los aprendizajes significativos con proposición experimental para su comprobación. Recuperado el 10 de Noviembre del 2012 de : http://www.cimm.ucr.ac. cr/ocs/files/conferences/1/ schedConfs/1/papers/2535/submission/review/2535-6949-1-RV.pdf

Pacheco, P., Villagran, S., Quiroz, E. (2013). Dinámica no lineal y rendimiento académico: verificación experimental e interpretación. Revista Internacional de Educación y Aprendizaje, Vol 1, $n^{\circ} 1,49-73$.

Pacheco, P., Villagrán, S., Guzmán, C. (2015). Estudio del campo emocional en aula y simulación de su evolución durante un proceso de enseñanza- aprendizaje para cursos de Ciencias. Revista Estudios Pedagógicos (aceptado para publicarse el primer semestre 2015)

Rodríguez, M. L. (2010). La teoría del aprendizaje significativo en la perspectiva de la psicología cognitiva. Barcelona: Octaedro

Serway, R. (1997). Física. Vol. I. México D.F.: McGraw-Hill. Interamericana Editores, S.A.

Spiegel, M. (2000). Estadística. Serie de Compendios Schaum's. Mexico D.F.: McGraw-Hill

Sprott, J.C. (2006). Chaos and Time - Series Analysis. New York: Oxford University Press

Schuster, H.G. (1995). Deterministic Chaos. Weinheim: Wiley-VCH Verlag

Stiglitz, J.(1987). The causes and consequences of the dependence of quality on Prices. Journal of 
Economic Literature, vol. 25, 1-48.

Treisman, U. (1992). Studying students studying calculus: A look at the lives of minority mathematics students in collage. College Mathematics Journal, 23(5), 362-372.

Varela, F., Thompson, E. y Rosch, E. (1997). De cuerpo presente. Barcelona: Gedisa

Zill, D. (1988). Ecuaciones Diferenciales con Aplicaciones. México D.F.: Ibero América

Ziv, J. y Lempel, A. (1977). A Universal Algorithm for Sequential Data Compression. IEEE Transactions on Information Theory, vol. IT-23, $\mathrm{n}^{\circ} 3,337-343$. 
